\title{
MODELO AUTO-REGRESSIVO APLICADO NA SIMULAÇÃO DE UM CANAL UWB
}

\author{
Fábio A. L. da Silva, ${ }^{1}$ \\ Leni J. de Matos $^{2}$ \\ Edson Cataldo ${ }^{3}$
}

\begin{abstract}
Resumo: Este artigo apresenta a modelagem e a simulação do canal rádio de banda ultralarga, com linha de visada entre transmissor e receptor, em um ambiente interno (indoor) da Universidade Federal Fluminense, baseado no modelo auto-regressivo da função seletividade em freqüência, medida no ambiente. Cinco métodos para implementar modelos auto-regressivos de segunda ordem são propostos, e os resultados da simulação do canal nesses modelos são comparados ao canal real, através dos parâmetros de pequena escala (banda de coerência, retardo médio e espalhamento de retardo). Os resultados obtidos são discutidos e, então, ajustes são propostos nos modelos de modo a se obter um melhor modelo do canal.
\end{abstract}

Palavras-chave: simulação, canal rádio UWB, banda ultralarga, modelo auto-regressivo.

Abstract: This paper introduces the modeling and the simulation of the ultra-wideband radio channel, with LOS between transmitter and receiver, in an indoor environment of the Federal Fluminense University, based in the autoregressive model of the frequency selectivity function, measured in the environment. Five methods to implement second order autoregressive models are proposed, and the results of the channel simulation in these models are compared to the real channel, through the small scale channel parameters (coherence bandwidth, mean delay and delay spread). The results obtained are discussed and, then, adjustments are proposed to the model in order to obtain a better model of the channel.

Keywords: simulation, UWB radio channel, ultra-wideband, autoregressive model.

\section{INTRODUÇÃO}

A tecnologia de comunicação em faixa ultralarga (UWB - Ultra-Wideband), cujos sinais têm largura de banda de freqüência, em valor absoluto, maior que $500 \mathrm{MHz}$, tornou-se uma solução importante para a implementação de sistemas de comunicações móveis de altas taxas de transmissão e de curto alcance, como, por exemplo, rede sem fio para dispositivos pessoais, também conhecida por wireless personal area network (WPAN) [1].

$\mathrm{O}$ canal por onde o sinal rádio se propaga exerce forte influência na comunicação em curso. Assim, é importante que se tenha um conhecimento profundo do mesmo para que se possam projetar melhores sistemas de comunicações. Para tal, é necessário que medidas sejam realizadas de forma a se determinar modelos que reproduzam, da melhor forma possível, o canal real. Estudos de modelos de canal rádio faixa-larga [2]-[4] serviram como base para que estudos recentes a respeito de modelos para canais UWB fossem desenvolvidos [5]-[7], os quais analisam as propriedades estocásticas da função resposta ao impulso $h(t ; \tau)$ (domínio tempo-retardo) ou a função seletividade de freqüência do canal $T(f ; t)$ (domínio freqüênciatempo), ambas representativas do mesmo canal, em diferentes domínios, sendo relacionadas por uma transformada de Fourier [8].

\footnotetext{
1 Universidade Federal Fluminense, Programa de Mestrado em Engenharia de Telecomunicações, e-mail:fadelia@oi.com.br.

2 Universidade Federal Fluminense, Programa de Mestrado em Engenharia de Telecomunicações, e-mail:lenijm@ig.com.br.

3 Universidade Federal Fluminense, Programa de Mestrado em Engenharia de Telecomunicações, e-mail:ecataldo@vm.uff.br.
} 
Neste artigo o modelo auto-regressivo (AR) é o aplicado por ser uma técnica que requer um pequeno número de parâmetros para modelar o canal rádio [4]. Este modelo AR estima uma determinada função, a partir de alguns valores conhecidos, neste caso, da função seletividade em freqüência $T(f ; t)$, que é obtida através da sondagem no domínio da freqüência [9]. Esta técnica emprega um analisador de rede vetorial acoplado a duas antenas, uma transmissora (TX) e outra receptora $(\mathrm{RX})$, separadas por uma determinada distância, para executar uma varredura discreta na banda de freqüência a ser analisada (960-1710 $\mathrm{MHz}$.

O artigo está distribuído da seguinte forma: a Seção II descreve como foram obtidas as medidas do canal rádio a ser simulado; a Seção III apresenta o modelo AR para simulação do canal rádio no domínio da freqüência, a Seção IV trata da modelagem, apresentando os quatros métodos baseados em [4] e o método baseado em [5]; a Seção V mostra os resultados das simulações e a Seção VI, as conclusóes.

\section{DESCRIÇÃO DO AMBIENTE DE MEDIDAS}

O ambiente usado foi o hall de entrada do Bloco D da Escola de Engenharia da Universidade Federal Fluminense, como esquematizado na Figura 1. Os dados foram obtidos por C. F. de Souza, em [10], em sua dissertação de mestrado, de acordo com a configuração descrita a seguir.

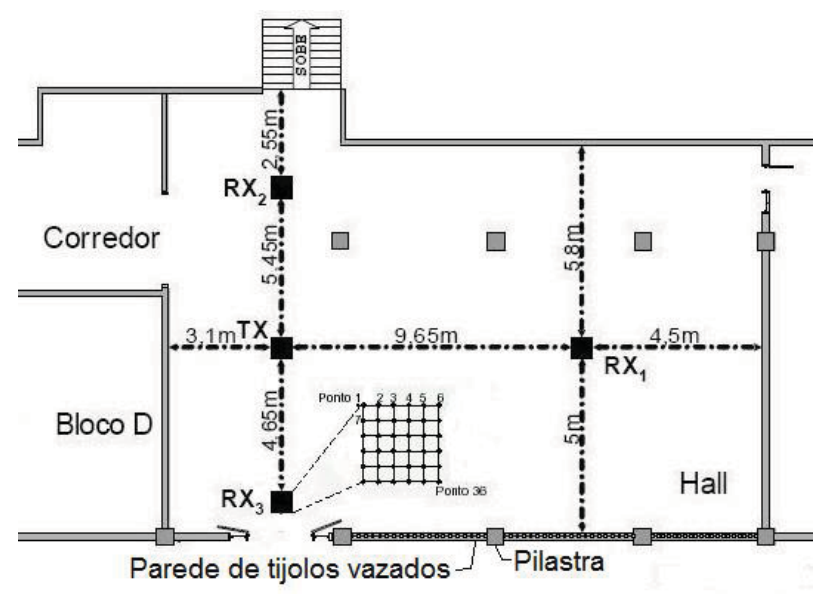

Fig. 1. Planta baixa do ambiente indoor medido.

O transmissor (TX) ficou fixo a uma distância aproximada de 3,1 m da parede do bloco
$\mathrm{D}$, enquanto o receptor foi deslocado para três regiōes distintas $\left(\mathrm{RX}_{1}, \mathrm{RX}_{2}\right.$ e $\left.\mathrm{RX}_{3}\right)$, com linha de visada (LOS - Line of Sight) para o transmissor. Cada regiāo de recepção é formada por um quadrado, de $75 \mathrm{~cm}$ de lado, representado por uma matriz quadrada $(6 \times 6)$, totalizando 36 pontos de medidas de $T(f, t)$. As entradas da matriz estão espaçadas de $15 \mathrm{~cm}$ uma da outra. Foi utilizada a técnica de varredura no domínio da freqüência para as medições, com as características apresentadas na Tabela I.

\section{Tabela I}

\section{Características da sondagem adotada}

\begin{tabular}{|c|c|c|c|}
\hline $\begin{array}{c}\text { Banda } \\
(\mathrm{MHz})\end{array}$ & $\begin{array}{c}\text { Freqüência } \\
\text { central } \\
(\mathrm{MHz})\end{array}$ & $\begin{array}{c}\text { Amostras por } \\
\text { varredura }\end{array}$ & $\begin{array}{c}\text { Tempo de } \\
\text { varredura } \\
(\mathrm{ms})\end{array}$ \\
\hline 750 & 1335 & 1601 & 696 \\
\hline
\end{tabular}

A sondagem do canal permitiu o levantamento de alguns dos parâmetros de pequena escala [10] para cada ponto de medição. São eles: banda de coerência, retardo médio e espalhamento de retardo. Tais parâmetros definem a dispersão temporal do sinal UWB no canal medido, sendo que a banda de coerência representa a menor separação em freqüência para a qual a função de autocorrelação de $T(f, t)$ corresponde, tipicamente, a um nível de $90 \%$ de correlação. É, portanto, a largura de faixa onde as componentes espectrais são afetadas de modo similar. Já o retardo médio e o espalhamento de retardo representam, respectivamente, a média e a variância do perfil de retardos de potência, $P_{b}(t ; \tau)$. Tal perfil é obtido através do quadrado do módulo da transformada inversa de Fourier de $T(f, t)$, no domínio da freqüência, ou seja: $P_{b}(t ; \tau)=\mathrm{F}^{-1}[$ $\left.|T(f ; t)|^{2}\right]$.

As funçōes $T(f, t)$ formam um processo estocástico. Observou-se, porém, que cada região de recepção $\left(\mathrm{RX}_{1}\right.$ a $\left.\mathrm{RX}_{3}\right)$ não apresentou significativa variabilidade dentro do curto tempo de varredura da banda de freqüência. Desta forma, assume-se que o processo estocástico é estacionário no sentido amplo e que cada função $T(f, t)$ foi medida em um único instante $t$. Com essas condiçốes, a autocorrelação, $R(k, 0)$, do processo $T(f, t)$, função somente da diferença entre as freqüências, $k$, é calculada por: 


$$
R(k)=\frac{1}{N} \sum_{i=1}^{N-k} T^{*}\left(f_{i}, t\right) \cdot T\left(f_{i-k}, t\right)
$$

onde $N$ é o número de amostras obtidas em uma varredura da banda de freqüência, $\mathrm{T}\left(f_{n}, \mathrm{t}\right)$ é o valor medido na freqüência discreta $f_{n}$, da banda medida, e $\mathrm{T}^{*}\left(f_{n}, \mathrm{t}\right)$ é o complexo conjugado de $\mathrm{T}\left(f_{n}, \mathrm{t}\right)$.

\section{MODELO AUTO-REGRESSIVO}

De acordo com o modelo AR, o valor de $T\left(f_{n}, t\right)$ é resultado de um processo auto-regressivo de ordem $p$, tal que:

$$
T\left(f_{n}, t\right)-\sum_{i=1}^{p} a_{i} T\left(f_{n}-i, t\right)=V\left(f_{n}, t\right)
$$

onde a função $V(f, t)$ é um ruído branco gaussiano complexo, cuja variância é dada por:

$$
\mathrm{s}_{V}{ }^{2}=R(0)-\sum_{i=1}^{p} a_{i} \cdot R(i)
$$

e os parâmetros $a_{i}$ da equação (2) são obtidos através da solução da equação de Yule-Walker:

$$
R(-m)-\sum_{i=1}^{p} a_{i} \cdot R(i-m)=0
$$

onde $m \geq 0$ e $p$ é a ordem do modelo AR.

Aplicando-se a transformada $\mathrm{Z}$ ao domínio de $f$ na equação (2), obtém-se pela relação entre $T(f, t)$ e $V(f, t)$ a função de transferência $G(Z)$, que é um filtro linear de $p$ pólos com resposta infinita ao impulso (IIR - Infinite Iimpulse Response). Assim, o modelo AR é capaz de gerar a função $\hat{T}(f, t)$, função estimada de $T(f, t)$, quando o filtro $G(Z)$ é excitado por um ruído branco gaussiano complexo, de variância dada pela equação (3). A Figura 2 mostra o diagrama de blocos referente a esse modelo.

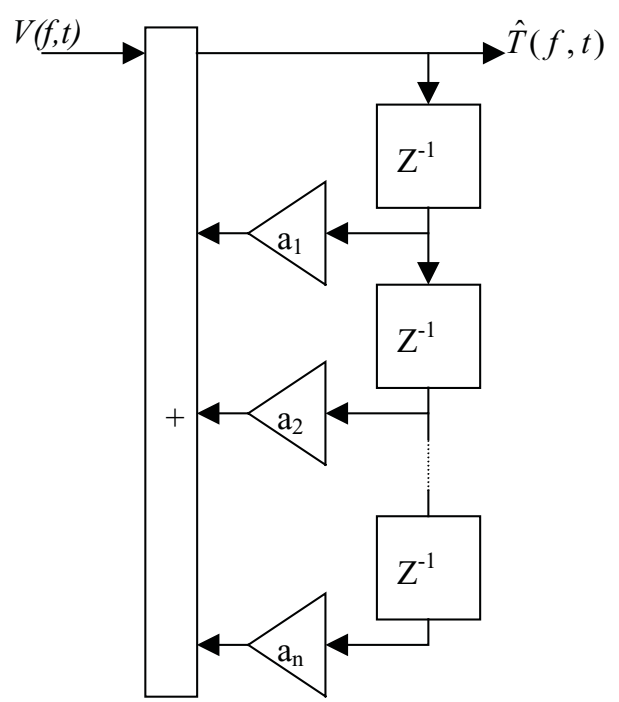

Fig. 2. Diagrama de blocos do modelo AR.

Neste artigo, a ordem escolhida para o modelo AR foi igual a 2, isto é, $p=2$. Tal escolha foi baseada nos critérios adotados por Howard e Pahlavan [4] e Ghassemzadeh et al. [5], que definiram esse valor para seus respectivos modelos. Ordens maiores foram aqui testadas, porém, a pequena melhora nos resultados não compensou o aumento da complexidade do modelo, em conseqüência do aumento da ordem. Nesse caso, a função de transferência do filtro IIR possui dois pólos $\left(p_{1}\right.$ e $\left.p_{2}\right)$ que, juntamente com a variância de $V(f, t)$, são os parâmetros a serem definidos no modelo AR do canal rádio UWB. Na Figura 3 é mostrada a disposição, no plano $Z$, dos pólos $p_{1} \mathrm{e}$ $p_{2}$, relativos à aplicação do processo auto-regressivo em cada uma das 36 funçôes $T(f, t)$, medidas na matriz da região de recepção $\mathrm{RX}_{3}$. Tais medidas serviram como base para simulação do canal nesta região. Uma observação importante é que as condiçôes iniciais do filtro $G(Z)$ influenciam na simulação do modelo $\mathrm{AR}$, como é mostrado nos gráficos da Figura 4, onde se vê os resultados da simulação para duas condições iniciais distintas: nulas e iguais a $T\left(f_{1}, t\right)$ e $T\left(f_{2}, t\right)$, medidos na primeira $\left(f_{1}\right)$ e na segunda $\left(f_{2}\right)$ freqüências no ponto 17 da regiāo $\mathrm{RX}_{3}$. Comparou-se a função $T(f, t)$ medida no referido ponto com as funçôes $\hat{T}(f, t)$ , resultantes da aplicação da função $V(f, t)$, obtida pela equação (2), na entrada de $G(Z)$, nas duas condições. 


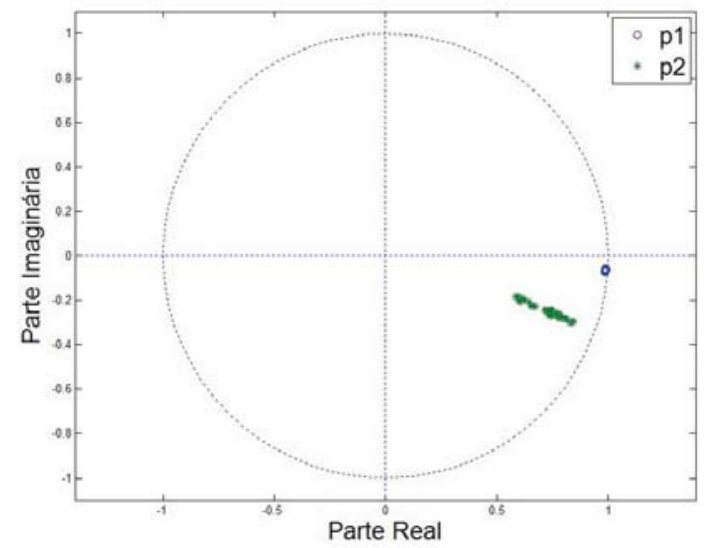

Fig. 3. Pólos $p_{1}$ e $p_{1}$, no plano Z, obtidos no modelo AR das medidas de $T(f, t)$ em $\mathrm{RX}_{3}$.

Constata-se que ambas as funções estimadas apresentam, no início da banda de freqüência, valores diferentes da função medida. A partir da freqüência de $1100 \mathrm{MHz}$, a função estimada, com condições iniciais nulas, é aproximadamente igual à função medida. Já a função estimada com condiçôes iniciais $T\left(f_{1}, t\right)$ e $T\left(f_{2}, t\right)$, aproxima-se da função medida em uma freqüência posterior, a partir da freqüência de $1400 \mathrm{MHz}$.

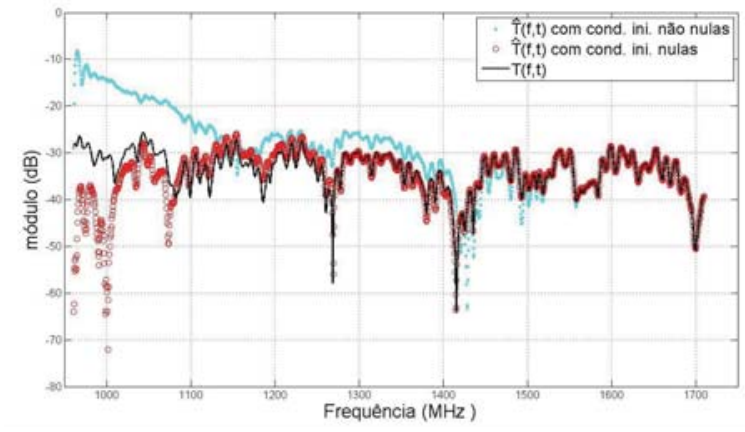

(a)

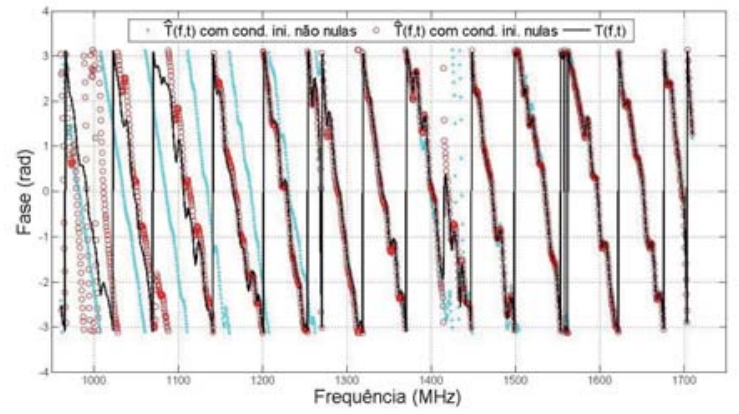

(b)

Fig. 4. Comparação entre $T(f, t)$ medida no ponto 17 de $\mathrm{RX}_{3}$ e $\hat{T}(f, t)$ gerada com condições iniciais não nulas e com condiçōes iniciais nulas. (a) módulo e (b) fase.
Em vista desses resultados, adotou-se, para este trabalho, condições iniciais nulas para o filtro IIR, do modelo AR, e a modelagem foi realizada na faixa de 1100 a $1710 \mathrm{MHz}$, na qual a função $\hat{T}(f, t)$ aproxima-se da função $T(f, t)$.

\section{MODELAGEM}

Para simular um canal rádio, baseado no modelo AR de $T(f, t)$, é necessário especificar um modelo para o módulo e a fase dos pólos e para a variância de $V(f, t)$, que está relacionada com a potência média recebida no ponto de medida. Embora, na realidade, esse modelo deva ser completamente probabilístico, a aproximação por um modelo determinístico pode, em alguns casos, ser usada devido às características dos sinais medidos. A seguir, serão discutidos cinco métodos para a modelagem dos pólos, partindo dos dados de módulo e fase dos pólos do modelo AR. Os métodos de (i) a (iv) foram especificados de maneira semelhante ao que foi empregado em [4] e o método (v) foi baseado nas especificações determinadas por [5].

Para os métodos de (i) a (iv), a fase de $p_{1}$ é fixa e igual à média das fases dos pólos $p_{1}$ e as demais características dos cinco métodos são descritos a seguir:

(i) os módulos de $p_{1}$ e $p_{2}$ e a fase de $p_{2}$ são variáveis aleatórias gaussianas, de média e variância obtidas através da distribuição dos respectivos pólos no plano Z;

(ii) a fase de $p_{2}$ é a média das fases dos pólos $p_{2}$ e os módulos de $p_{1}$ e $p_{2}$ são variáveis aleatórias gaussianas como em (i);

(iii) o módulo e a fase de $p_{2}$ são iguais aos valores médios de módulo e da fase dos pólos $p_{2}$ e o módulo de $p_{1}$ é uma variável aleatória gaussiana como em (i);

(iv) os módulos de $p_{1}$ e $p_{2}$ e a fase de $p_{2}$ são iguais às médias das distribuições dos respectivos pólos no plano $\mathrm{Z}$; e

(v) os módulos de $p_{1}$ e $p_{2}$ são variáveis aleatórias com distribuição Weibull e as fases de $p_{1}$ e $p_{2}$ são variáveis aleatórias gaussianas.

Assumiu-se, para todos os métodos, que a variância do ruído branco, entrada do filtro $G(Z)$, é igual a: 


$$
\mathrm{S}_{V}{ }^{2}=\frac{R(0)}{g(n) \otimes g *(-n)}, n=0
$$

onde $R(0)$ representa a potência média recebida num dado ponto da região $\mathrm{RX}_{3}$, que é modelada como uma função lognormal, de média e variância obtidas por intermédio das 36 medidas de $T(f, t)$, na referida região, e $g(n)$ é a resposta ao impulso do filtro IIR do modelo AR construído por cada método.

De acordo com [4], o modelo para potência média recebida, que foi adotado neste estudo, é adequado para modelar o canal rádio em uma pequena área, como a região $\mathrm{RX}_{3}$. Entretanto, é possível gerar um modelo AR capaz de simular $T(f, t)$ em função da distância TX-RX, se for adotado um modelo para potência média recebida mais complexo, como em [5], que represente a atenuação do sinal em função da distância.

\section{SIMULAÇŌES E RESULTADOS}

Após gerar 100 funções $\hat{T}(f, t)$, para cada um dos cinco métodos utilizados, foi feita a comparação dos parâmetros de curta escala: banda de coerência, retardo médio e espalhamento de retardo. Esses parâmetros foram obtidos tanto para as funções $\hat{T}(f, t)$ estimadas como para as funções $T(f, t)$ medidas.

As Figuras 4, 5 e 6, respectivamente, mostram comparações entre os gráficos das funções distribuições de probabilidade da banda de coerência, retardo médio e espalhamento de retardo, para o canal medido e para o simulado, através do emprego dos cinco métodos descritos anteriormente.

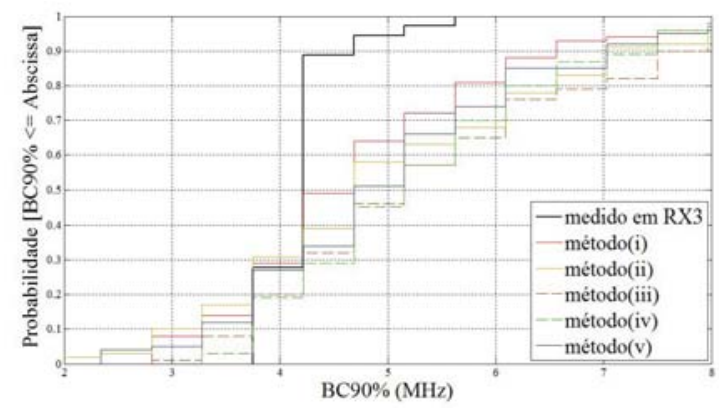

Fig. 4. Função distribuição de probabilidade para a banda de coerência de 90\% (BC90\%) dos canais simulados e medido.

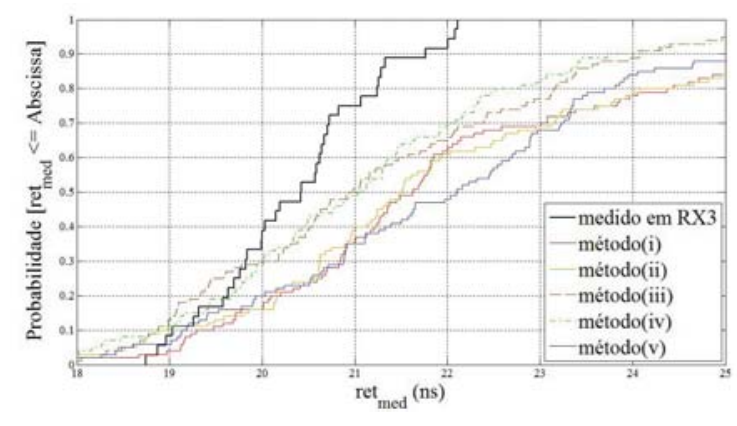

Fig. 5. Função distribuição de probabilidade para o retardo médio $\left(\right.$ ret $\left._{\text {med }}\right)$ dos canais simulados e medido.

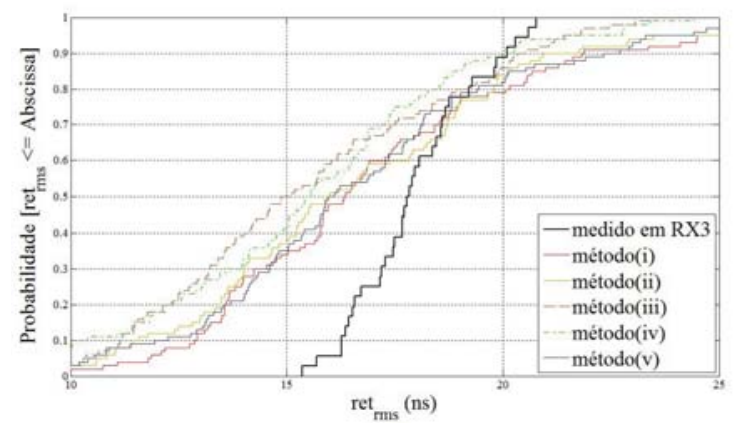

Fig. 6. Função distribuição de probabilidade para o espalhamento de retardo ( ret $_{\mathrm{rms}}$ ) dos canais simulados e medido.

Observa-se, nestes gráficos, que a faixa de variação dos parâmetros do canal rádio medido está contida na faixa de variação dos parâmetros do canal simulado pelos cinco métodos adotados. Portanto, todos os métodos empregados foram capazes de gerar modelos compatíveis ao canal medido.

Uma outra ferramenta ainda utilizada, para comparar o canal rádio simulado com o real, foi verificar se as autocorrelações $R(k)$ das 36 funções $T(f, t)$ encontram-se dentro do intervalo máximo e mínimo das autocorrelações, $\hat{R}(k)$, das funçōes estimadas $\hat{T}(f, t)$, ou seja, no intervalo de confiança da autocorrelação. Como exemplo, 500 funçôes $\hat{T}(f, t)$ foram geradas pelo método (iv), o menos complexo entre os cinco apresentados. Então, verificou-se, conforme mostrado na Figura 7, que os módulos das autocorrelações das 36 funções $T(f, t)$, da região $\mathrm{RX}_{3}$, encontram-se no interior do intervalo das autocorrelaçōes, $\hat{R}(k)$, das funções $\hat{T}(f, t)$. Portanto, este método pode gerar funções $\hat{T}(f, t)$ que apresentam autocorrelaçōes compatíveis com as autocorrelações das funções $T(f, t)$.

Várias outras ferramentas poderiam ter sido usadas, partindo do mesmo princípio, como usar outros métodos. Além disso, seria necessário um 
estudo de convergência para determinar o número de funçôes $\hat{T}(f, t)$ que deveriam ser geradas. Porém, o objetivo aqui, é apenas mostrar a possibilidade de mais uma ferramenta a ser utilizada para possibilitar a comparação entre os canais real e simulado que, nesse caso, mostrou um ótimo comportamento.

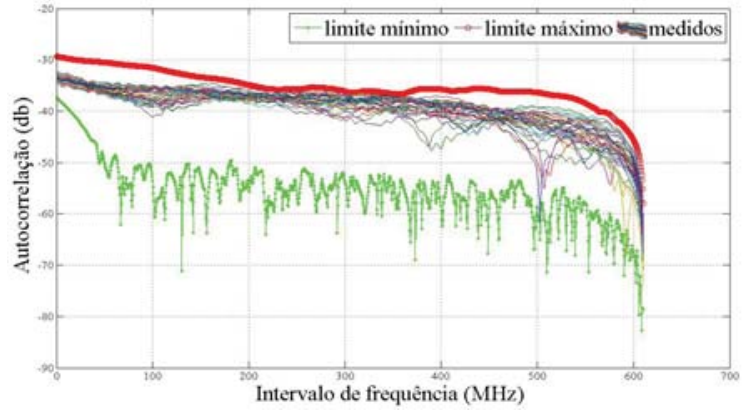

Fig. 7. Autocorrelação das 36 funçôes medidas em $\mathrm{RX}_{3}$, limites máximo e mínimo da autocorrelação das funções simuladas pelo método (iv).

\section{CONCLUSŌES}

A modelagem do canal rádio, em um ambiente indoor escolhido, foi proposta e os resultados simulados foram comparados com os dados experimentais. A modelagem proposta foi baseada em trabalhos anteriores, porém ajustes, em termos de condições iniciais do filtro e faixa de variação dos pólos $p_{1}$ e $p_{2}$, foram realizados a fim de adequar o modelo AR aos resultados medidos disponíveis.

Todos os cinco métodos empregados na modelagem dos pólos foram capazes de gerar funçôes estimadas $\hat{T}(f, t)$ que apresentaram mesmas características das funções $T(f, t)$ medidas, entretanto, em termos de complexidade computacional, o método (iv) mostrou-se mais adequado uma vez que foi gerado um modelo AR de pólos determinísticos.

Logo, o uso do modelo AR, com os devidos ajustes, como diminuir a banda de freqüência simulada em relação à banda medida, mostrou-se adequado para modelar o canal rádio, com baixa complexidade e pequena quantidade de parâmetros.

Objetivando melhorar a modelagem do canal, ajustes ainda podem ser feitos, destacando-se dentre eles:

- empregar um número maior de medidas de $T(f, t)$ e um modelo de atenuação do sinal com a distância, a fim de aumentar a complexidade do ambiente e obter uma faixa maior de valores para os parâmetros do modelo;

- propor outras funções distribuições de probabilidade para os pólos do modelo AR, empregando o Princípio da Máxima Entropia [11] na determinação dessas distribuições de probabilidade, uma vez que esse Princípio é bem aplicado nesse caso, pois a quantidade de dados experimentais é pequena;

- discutir o problema gerado pela resposta inicial do filtro IIR, impedindo que a função $T(f, t)$ seja estimada em toda a banda de frequência medida. Nesse caso, duas direçôes podem ser tomadas: detectar as condições iniciais do filtro que ajustem melhor a resposta inicial ou empregar um modelo AR modificado.

\section{REFERÊNCIAS}

[1] G. R. Aiello e G. D. Rogerson, "Ultra-wideband Wireless Systems", IEEE Microwave Magazine, v. 4, pp. 36-47, Junho 2003.

[2] A. A. M. Saleh e R. A. Valenzuela, "A statistical model for indoor multipath propagation", IEEE J. Select. Areas Commun., v. 5, pp. 128-137, Fevereiro 1987.

[3] H. Hashemi, "The indoor radio propagation channel”, Proc. IEEE, v. 81, pp. 943-968, Julho 1993.

[4] S. Horward e K. Pahlavan, "Autoregressive Modeling of Wide-Band Indoor Radio Propagation", IEEE Transaction on Commun., v. 40, pp. 1540-1552, Setembro 1992.

[5] S. S. Ghassemzadeh, R. Jana,V. Tarokh, C. W. Rice e W. Turin, "Measurement and Modeling of an Ultra-Wide Bandwidth Indoor Channel", IEEE Transactions on Comm., v. 52, pp. 17861796, Outubro 2004.

[6] D. Cassioli, M. Z. Win e A. Molisch, "The ultra-wide bandwidth indoor channel: from statistical model to simulations", IEEE J. Select. Ar- 
eas Commun., v. 20, n. 6, pp. 1247-1257, Agosto 2002.

[7] A. F. Molisch, J. R. Foerster e M. Pendergrass, "Channel models for ultra-wideband personal area networks", IEEE Wireless Commun. Mag., v. 10, n. 6, pp. 14-21, Dezembro 2003.

[8] D. Parsons, The Mobile Radio Propagation Channel, New York: John Wiley \& Sons, 1992.

[9] S. Haykin, Adaptive Filter Theory, Upper Saddle River, NJ:Pertince-Hall,1996.
[10] C. F. de Souza, Análise da dispersão temporal de canais de banda ultralarga (UWB) através de medidas realizadas em ambientes internos e externos, Dissertação de Mestrado, Universidade Federal Fluminense, Niterói, 2006.

[11] J. N. Kaput e H. K. Kesavan, Entropy optimization principles with applications, Academic Press Inc., 1992. 